Portland State University

PDXScholar

$5-24-2019$

\title{
Teaching Geometrical Optics by Modeling Fluidic Lenses Formed by Water-Walking Insects
}

Erin Woodcock

Portland State University

Follow this and additional works at: https://pdxscholar.library.pdx.edu/honorstheses

Let us know how access to this document benefits you.

Recommended Citation

Woodcock, Erin, "Teaching Geometrical Optics by Modeling Fluidic Lenses Formed by Water-Walking Insects" (2019). University Honors Theses. Paper 761.

https://doi.org/10.15760/honors.778

This Thesis is brought to you for free and open access. It has been accepted for inclusion in University Honors Theses by an authorized administrator of PDXScholar. Please contact us if we can make this document more accessible: pdxscholar@pdx.edu. 
Teaching Geometrical Optics by Modeling Fluidic Lenses Formed by Water-Walking Insects

by

\title{
Erin Woodcock
}

An undergraduate honors thesis submitted in partial fulfillment of the

\author{
requirements for the degree of \\ Bachelor of Arts/Science \\ in University Honors \\ and
}

Physics

\author{
Thesis Advisor \\ Dr. Ralf Widenhorn
}

Portland State University

2019 


\begin{abstract}
When a water-walking insect sits atop the surface of a fluid, its legs create shadows that are disproportionately large and are surrounded by bright rings of light. While these shadows are prominent, their cause is not easily perceptible to an observer's eye. The phenomenon is the result of the formation of fluidic lenses caused by the insect legs deforming the surface of the fluid. In this work, an experimental setup is created to emulate the conditions under which these shadows are formed and explore the properties of the shadows and the deformed fluid's surface. The setup is inexpensive, consisting of an LED lamp and a 3D printed apparatus attached to a ring stand. The methods used in this work can be used to create lab activities for physics students to help them to better understand geometrical optics as well as the interactions between solids, fluids, and air.
\end{abstract}

\title{
INTRODUCTION AND BACKGROUND
}

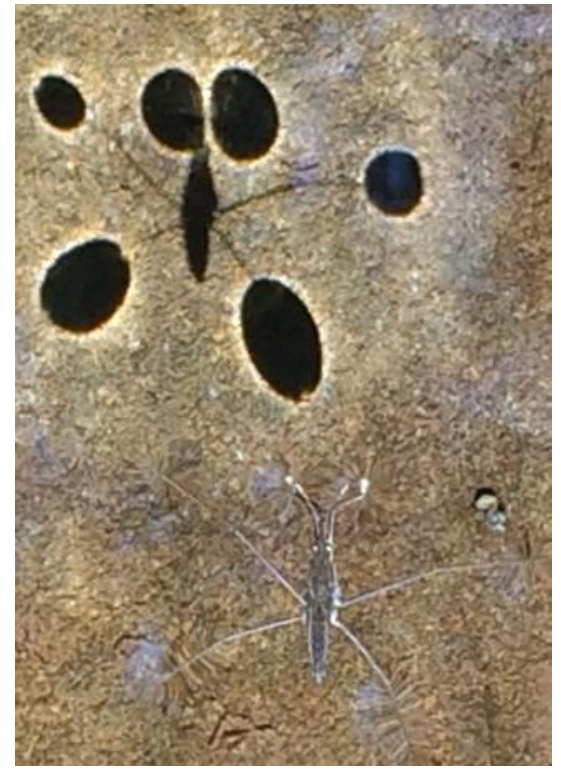

Fig. 1: A water strider sitting stationary on the surface of a body of water and the shadows it casts on the ground below it.

The modeling of natural phenomena in a physics classroom is quite possibly the most tangible way to demonstrate to students the utility of physics knowledge and research. Geometrical optics in particular can be challenging for physics students, and there is a clear need for new and innovative classroom experiments to ameliorate students' understanding of the subject. One of the best ways to do so is to present a natural, optical phenomenon with which students are familiar and challenge them to understand and describe that phenomenon under the lens of geometrical optics. One such natural phenomenon could be the lens formation caused by the surface tension interaction between a fluid and a water-walking insect's leg, which produces the shadows ringed by light observed in Fig. 1, and raises a host of questions about both the physics of surface tension as well as geometrical optics. The question, then, is how can the optical properties of fluidic lenses formed by an insect leg be explored in an undergraduate physics classroom? Before answering this question, the physics of surface tension and optical properties of the fluidic lenses must be fully understood. Each phenomenon will be explored in detail. 


\section{Surface Tension Physics and Fluidic Lens Formation}

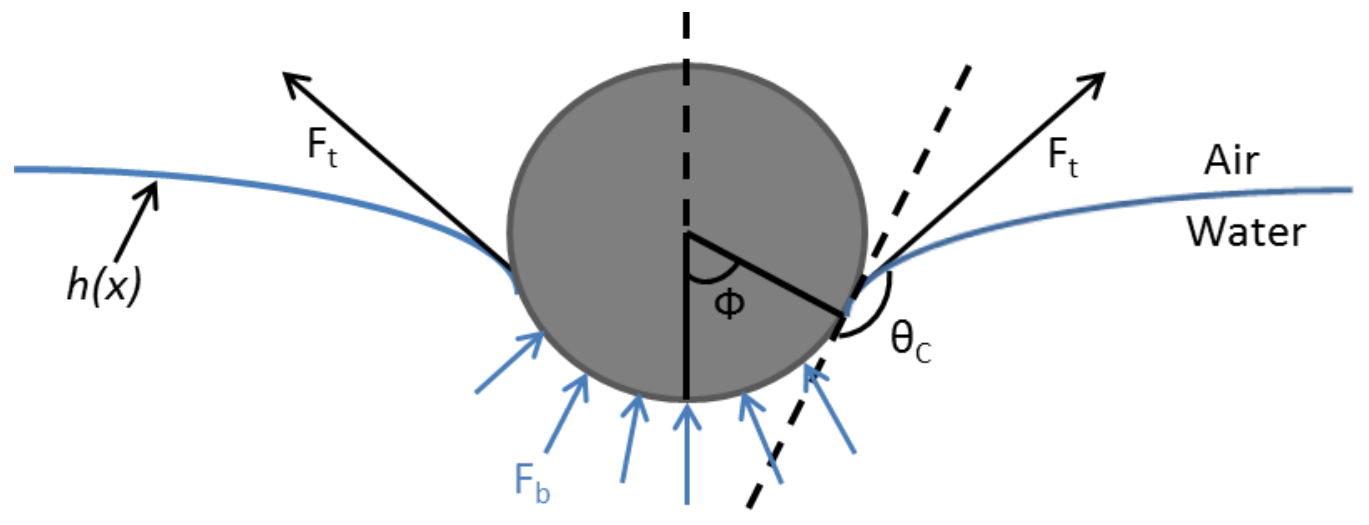

Fig. 2: Diagram of the interface between a cylindrical object (such as an insect leg) and a fluid, showing the forces required to keep the insect's leg from penetrating the water surfae. ${ }^{1,2}$

In order for an insect to sit atop the surface of a fluid, its full weight, $m g$ (where $m$ is the insect's mass and $g$ is the gravitational constant), must be supported by the fluid. This is accomplished by a lift force: a combination of buoyancy force and the vertical component of the surface tension force (see Fig. 2). ${ }^{1,2}$ The buoyancy force $F_{b}$ is given by:

$$
F_{b}=\rho_{f} V_{f} g,
$$

where $\rho_{f}$ is the fluid density and $V_{f}$ is the volume of fluid displaced by the insect leg. In other words, in accordance with Archimedes' principle, the buoyancy force is equivalent to the weight of the fluid displaced. If the insect leg were fully submerged in the fluid, the buoyancy force would be the only force needed to be accounted for in order to counteract the weight of the strider. However, thanks to surface tension force, the interacting forces become slightly more complicated. Surface tension force $F_{t}$ is given by:

$$
F_{t}=\sigma s
$$

where $\sigma$ is the surface tension of the fluid and $s$ is the circumference of the portion of the object that is in contact with the fluid. The vertical component of the surface tension force is then given by:

$$
F_{t, z}=\sigma s \sin \left(\theta_{c}\right)
$$

where $\theta_{c}$ is the contact angle between the insect leg and the surface of the fluid (see Fig. 2). The contact angle is a property of the material that interacts with the fluid, defined by the angle created by the interface between the fluid, air, and the material. Materials with contact angles larger than $90^{\circ}$ are said to be hydrophobic, while contact angles smaller than $90^{\circ}$ are said to be hydrophilic. Most water-walking insect legs are superhydrophobic; for example, the contact angle of a water strider's leg has been measured to be $168.8^{\circ}$, just $11.2^{\circ}$ smaller than the largest possible contact angle of $180^{\circ} .^{3}$

The combination of the buoyancy force and the surface tension force counterbalances the weight of the insect such that it is able to sit comfortably on the surface of a fluid. Because the insect leg does not 
break the surface tension of the fluid, it deforms the fluid's surface. The approximate shape of the fluid's surface is given by the following relation:

$$
h\left(x, \Phi, \theta_{c}\right)=B\left(\Phi, \theta_{c}\right) e^{-\frac{1}{c} x},
$$

where $h$ is the depth of the dimple formed by the insect leg, $x$ is the position on the $\mathrm{x}$ axis where the center of the insect leg is the origin, $\Phi$ is the submerge angle, given by:

$$
\Phi=\sin ^{-1}\left(\frac{x_{0}}{R}\right)
$$

in which $R$ is the radius of the insect leg, $x_{0}$ is the water take-off point, and $B$ is given by:

$$
B\left(\Phi, \theta_{c}\right)=-c e^{\frac{1}{c} R \sin \theta_{c}} \tan \left(\theta_{c}+\Phi-\pi\right)
$$

in which $c$ is given by:

$$
c=\sqrt{\frac{\sigma}{\rho g} \frac{1}{\left(1+m_{L}^{2}\right)^{\frac{3}{2}}}},
$$

in which $m_{L}$ is the mass of the insect leg. ${ }^{4,5,6}$

The deformation of the fluid's surface results in the creation of a fluidic lens, with a focal length $f$ given by:

$$
f=\frac{2 \sigma}{\Delta n \Delta P},
$$

where $\Delta n$ is the difference in indices of refraction between air and the fluid and $\Delta P$ is the difference in internal and external pressures of the fluid's surface, caused by the weight of the insect leg. ${ }^{7}$ This relation comes from a combination of the Young-Laplace equation for capillary pressure:

$$
\Delta P=\frac{2 \sigma}{R_{C}}
$$

where $R_{C}$ is the radius of curvature of the fluidic lens, and the lens-maker's equation:

$$
\frac{1}{f}=\Delta n\left[\frac{1}{R_{1}}-\frac{1}{R_{2}}\right]
$$

where $R_{1}$ and $R_{2}$ are the radii of curvature of the lens surface closer to the light source and farther from the light source, respectively. Substituting $R_{C}$ from Eq. 9 for $R_{l}$ and $\infty$ for $R_{2}$ (as the opposite end of the lens can be approximated as a plane, at the interface between the fluid and the ground) results in Eq. 8 above. 


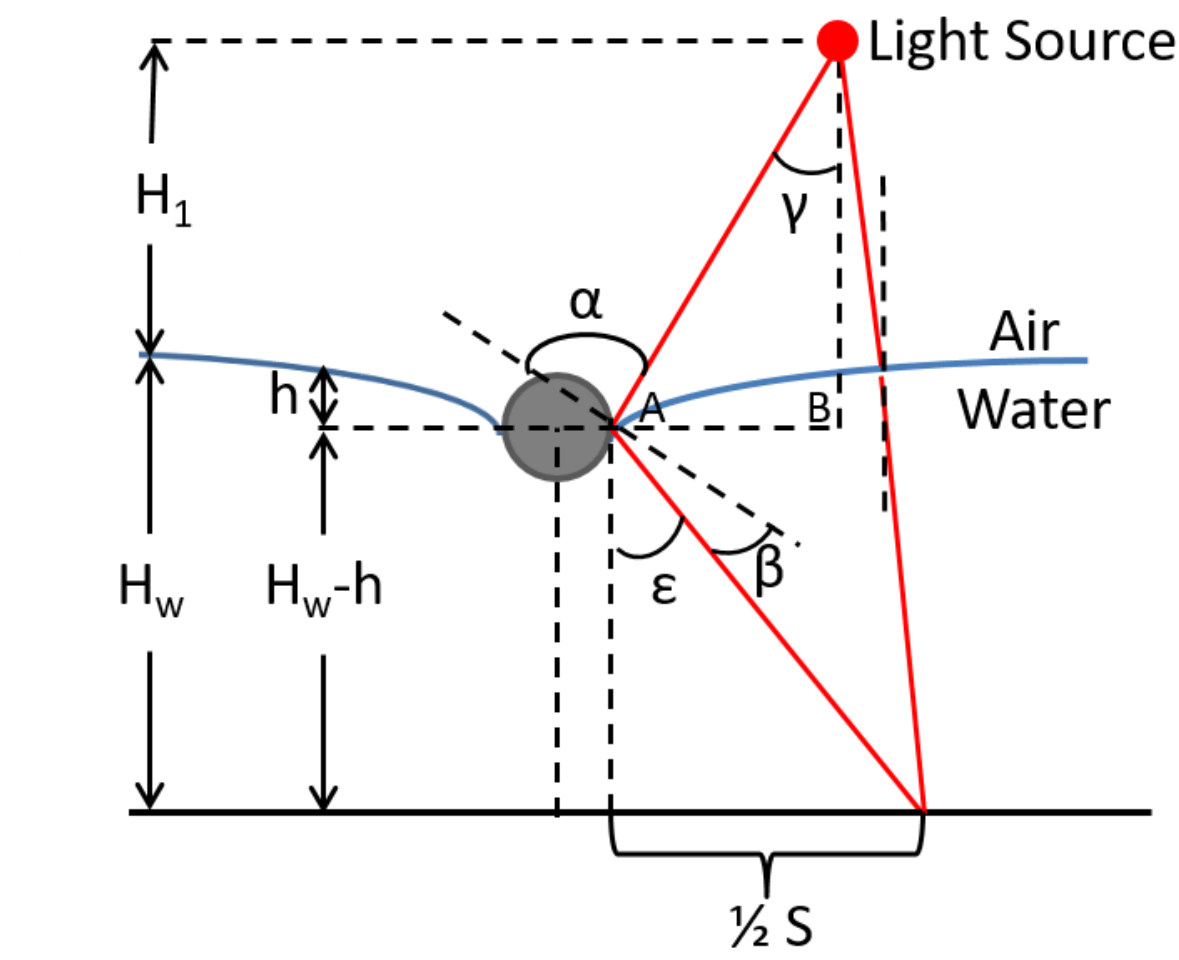

Fig. 3: Light refraction at the air/water interface of the dimple created by an insect leg. ${ }^{4,5,6}$

The formation of the shadows that appear on the ground below the insect is a product of the fluidic lens formation caused by the deformation of the water's surface by the insect legs, as described in the previous section. The optical properties of the fluidic lens are diagrammed in Fig. 3 , where $H_{l}, H_{w}$, and $h$ are the distance between the light source and the water's surface, the distance between the water's surface and the ground, and the distance between the water's surface and the center of the insect leg, respectively. ${ }^{4,5} \alpha$ and $\beta$ represent the angle of incidence and angle of refraction of the light, respectively, and $S$ is the width of the shadow cast on the ground below the insect. When incident light comes into contact with the water's surface, it is refracted in accordance with Snell's law:

$$
n \sin (\alpha)=n^{\prime} \sin (\beta)
$$

where $n$ is the index of refraction of air and $n$ ' is the index of refraction of the fluid. From Fig. 3, it can be determined that $\gamma$ is given by:

$$
\gamma=\tan ^{-1}\left(\frac{A B}{H_{1}+h}\right)
$$

where $A B$ is the horizontal distance between the edge of the insect leg and the light source. From Fig. 3, one can see that $\varepsilon$ is then given by:

$$
\varepsilon=90^{\circ}-\beta-\gamma .
$$


From this information, the total width of the shadow cast by the leg can then be determined:

$$
S=2\left(H_{w}-h\right) \tan \varepsilon
$$

(here the radius of the insect leg is ignored, assuming that $S \gg R$ ). Equation 4 predicts that the resulting effect is a shadow that is much larger than the insect leg cast on the ground below, with a bright ring around the shadow where the light rays converge. The shadow is caused by the deflection of the light rays to the point where the bright ring is formed. Through these relations, it can be determined that the size of the shadow cast by the insect is largely governed by the index of refraction of the fluid upon which it stands, the angle of incidence of the light as it strikes the dimple formed by the strider's leg, the depth of the fluid, and the depth of the dimple. 


\section{EXPERIMENTAL}

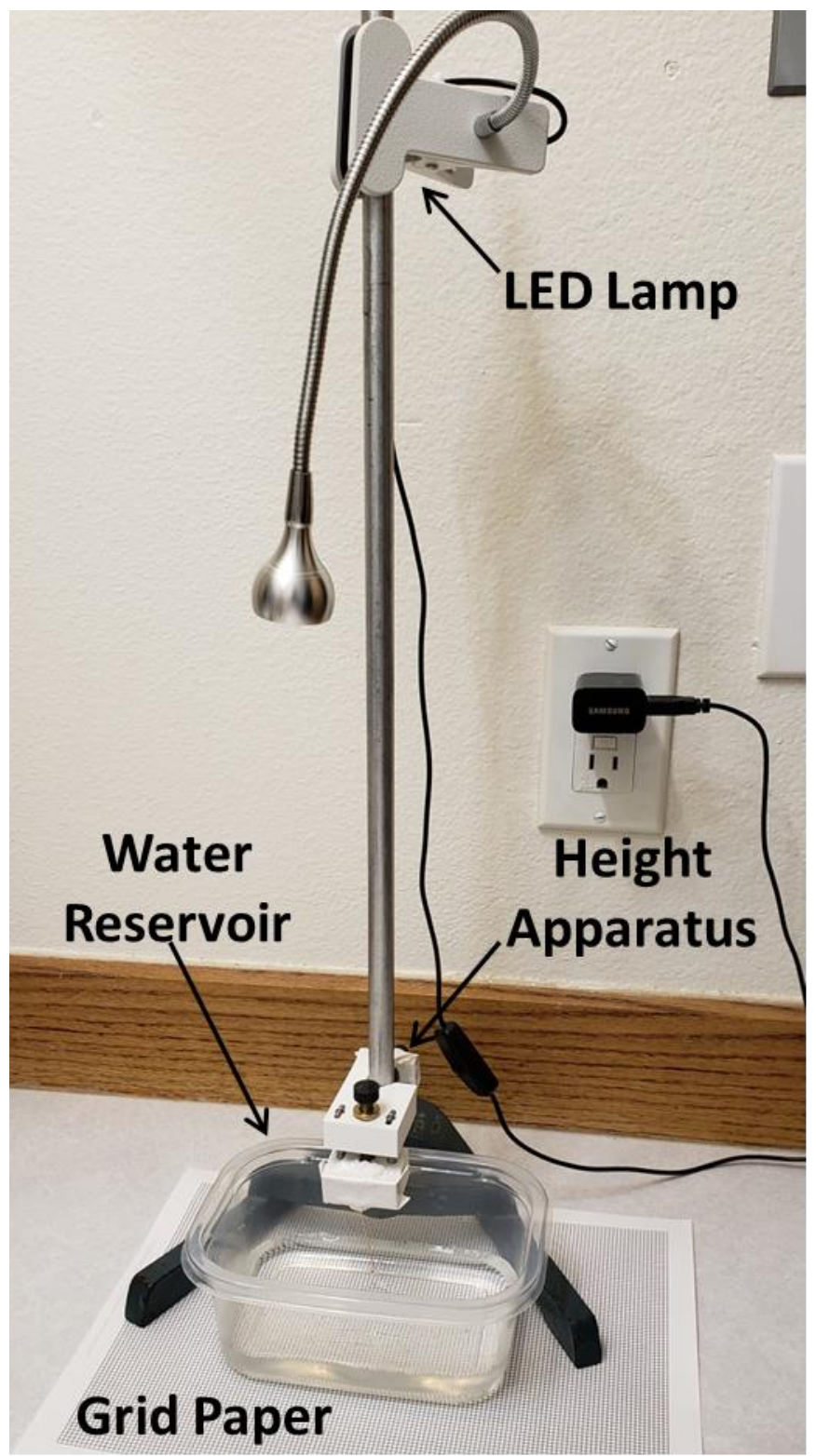

Fig. 4: The experimental setup, showing the ring stand, lamp, height apparatus, water reservoir, and grid paper.

In order to test the theoretical model discussed in the previous section, a variety of experiments were designed to emulate the conditions under which the shadows are observed naturally. A white LED lamp was used as a point source of light, and a small plastic container was used as a water reservoir. In one experiment, the insect leg was simulated using a $0.27 \mathrm{~mm}$ radius copper wire. The part of the wire that makes contact with the surface of the water was made to be $3 \mathrm{~mm}$ long. In addition to using a bare copper wire, a hydrophobic silicone spray and car wax were used to increase the contact angle of the wire. The contact angles of these materials were measured by applying the material to a flat ceramic surface, allowing the material to cure per recommendations for the material, then placing a water droplet on the 
surface and measuring the angle between the coated ceramic surface and the edge of the water droplet (see Fig. 6). The same method was used to experimentally measure the contact angle of Teflon (using a Teflon coating spray) and copper (using a copper weight).

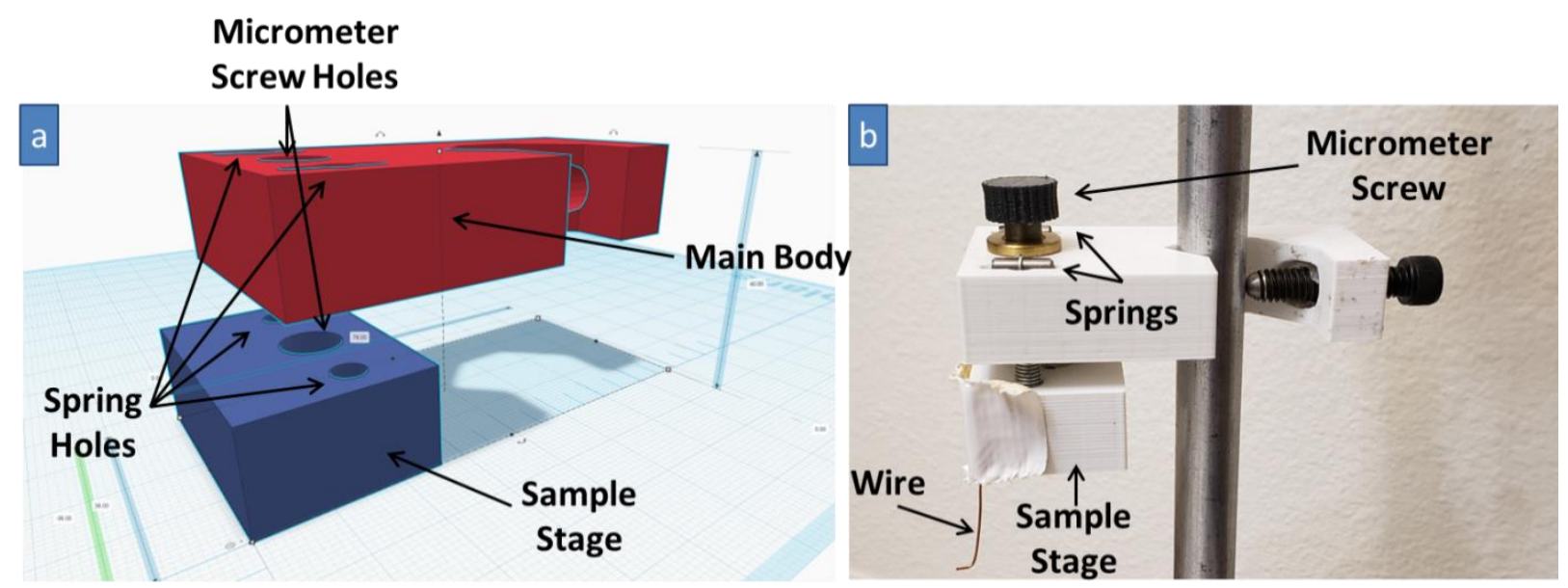

Fig. 5: a) The Tinkercad schematic of the apparatus designed to raise and lower the wire leg into the water, b) the actual 3D printed apparatus as used for experimentation.

The wire legs were raised and lowered into the water using an apparatus that was designed in Tinkercad and 3D printed for this purpose (see Fig. 5). A micrometer screw, when turned clockwise, was used to push the sample stage down, while the springs were used to provide tension and allow the stage to be raised as the micrometer screw was turned counter-clockwise. One full turn of the micrometer screw was found to change the height of the sample stage by $200 \mu \mathrm{m}$. The apparatus and lamp were attached to a ring stand (see Fig. 4), the wire leg was taped to the sample stage, and it was lowered to where the simulated $3 \mathrm{~mm}$ leg was just above the water's surface. The wire leg was then incrementally lowered using the apparatus and the resulting shadow cast on the lower surface of the water reservoir was measured using grid paper with a grid spacing of $1 \mathrm{~mm}$ placed below the reservoir. The shadows were measured in this fashion until the wire leg fully penetrated the water's surface, resulting in the disappearance of the shadow.

The apparatus and the method described above were used in a variety of experiments designed to explore several different aspects of the physical and optical properties of insect water-walking. First, the relationship between maximum dimple depth and contact angle was explored by coating wires of the same radius with silicone spray and car wax and comparing the results to that of a bare copper wire. In another experiment, the role of wire radius in dimple depth and shadow width was investigated using magnet wires of radius $0.29 \mathrm{~mm}$ and $0.59 \mathrm{~mm}$, and Teflon wires of radius $0.65 \mathrm{~mm}$ and $0.74 \mathrm{~mm}$. Another experiment examined how the width of the shadow changed with increasing water depth, using the $0.65 \mathrm{~mm}$ radius Teflon wire and water depths between $15 \mathrm{~mm}$ and $35 \mathrm{~mm}$ at intervals of $5 \mathrm{~mm}$. Finally, the dimple depths and shadow widths formed in fluids of varying refractive indices and surface tensions were compared using water, isopropyl alcohol, ethyl alcohol, vegetable oil, and canola oil. 


\section{RESULTS AND DISCUSSION}

\section{Contact Angles}
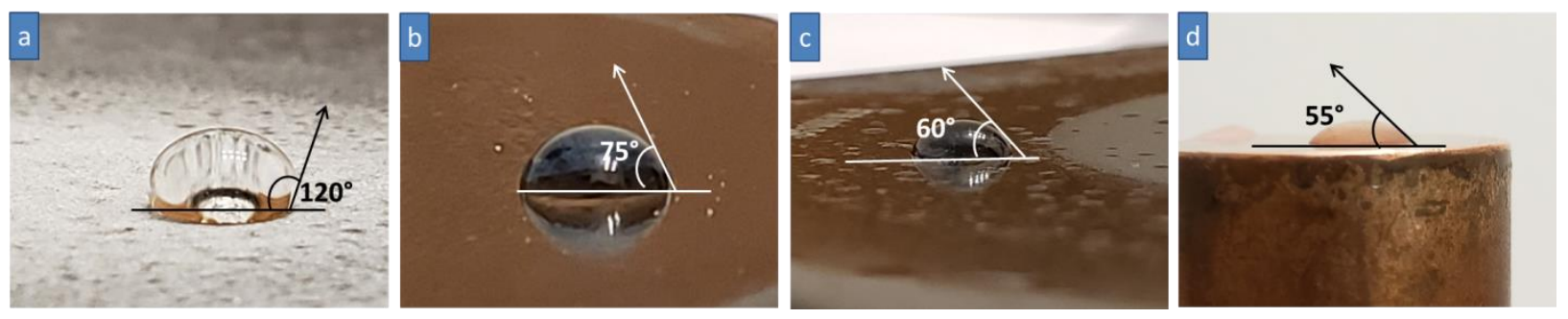

Fig. 6: Measured contact angles between water and: a) Teflon, b) silicone spray, and c) car wax, and d) copper.

The contact angles between water and the materials used for this experiment were measured to be the following: $120^{\circ}$ for Teflon, $75^{\circ}$ for the silicone spray, $60^{\circ}$ for the car wax, and $55^{\circ}$ for bare copper (see Fig. 9). This means that of these materials, Teflon is the most hydrophobic, while the bare copper wire is the most hydrophilic.

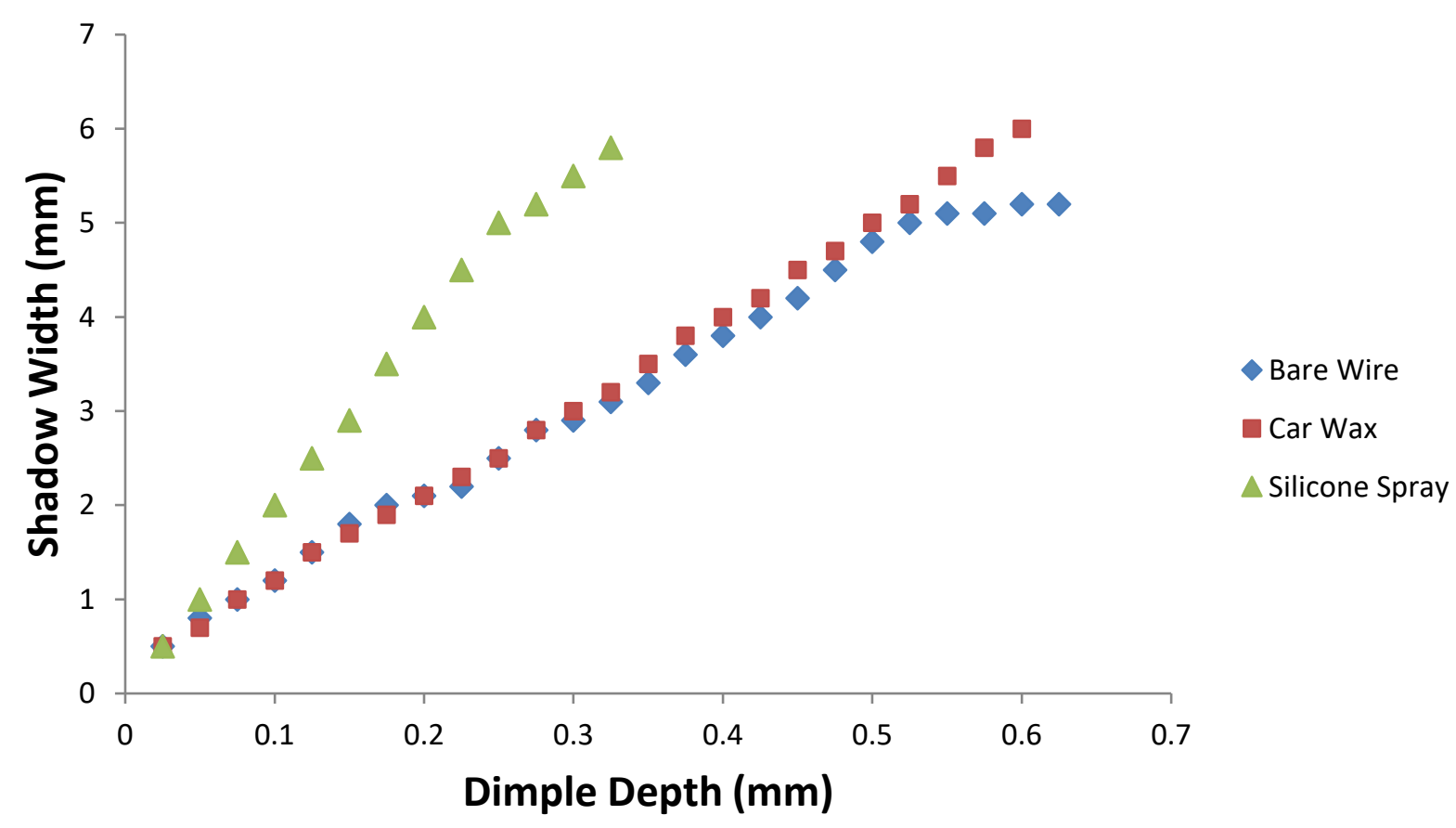

Fig. 7: Shadow width vs. dimple depth for each of the materials at a water depth of $15 \mathrm{~mm}$.

It was found that the bare wire created the deepest dimple on the water's surface, with a maximum dimple depth of $0.625 \mathrm{~mm}$, and the silicone spray coated wire created the shallowest dimple, with a maximum dimple depth of $0.325 \mathrm{~mm}$ (see Fig. 7). The car wax coated wire was found to create a maximum dimple depth of $0.6 \mathrm{~mm}$. These results confirm that increasingly hydrophilic materials were able to penetrate the water surface more deeply than hydrophobic materials. Equation 14 predicts that 
shadow width should generally increase linearly with increasing dimple depth $h$ (with slight deviations being accounted for by the $\gamma$ term in Eqs. 12 and 13), which was also the observed trend in Fig. 7.
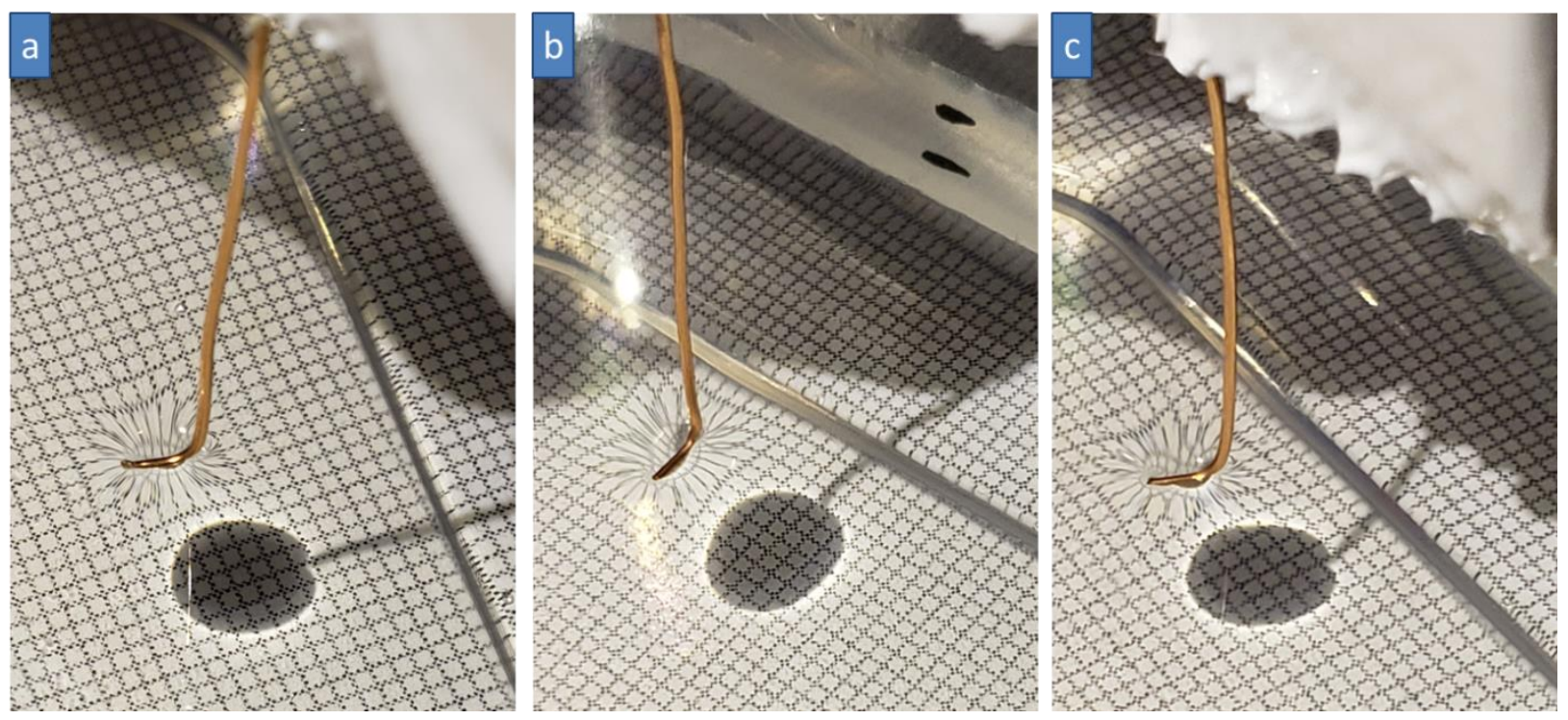

Fig 8: The maximum shadow widths observed for the a) bare wire, b) car wax coated wire, and c) silicone spray coated wire at a water depth of $15 \mathrm{~mm}$.

With a water depth of $15 \mathrm{~mm}$ and a $15 \mathrm{~cm}$ distance from the LED to the water's surface, it was found that the maximum shadow size for the silicone spray and car wax coated wires was $6 \mathrm{~mm}$ (see Fig. 8), while the maximum shadow size for the bare wire was observed to only be $5.2 \mathrm{~mm}$. These results suggest that in general, the contact angle affects the maximum dimple depth much more than the maximum shadow size, though the effect of contact angle on shadow size may become more significant with increasing hydrophilia of materials. Moreover, because more hydrophilic materials create deeper dimples and tend to maintain the same maximum shadow size as the other materials, the incremental changes in shadow size with dimple depth for more hydrophilic materials were generally smaller than those for more hydrophobic materials. 


\section{Wire Radius}

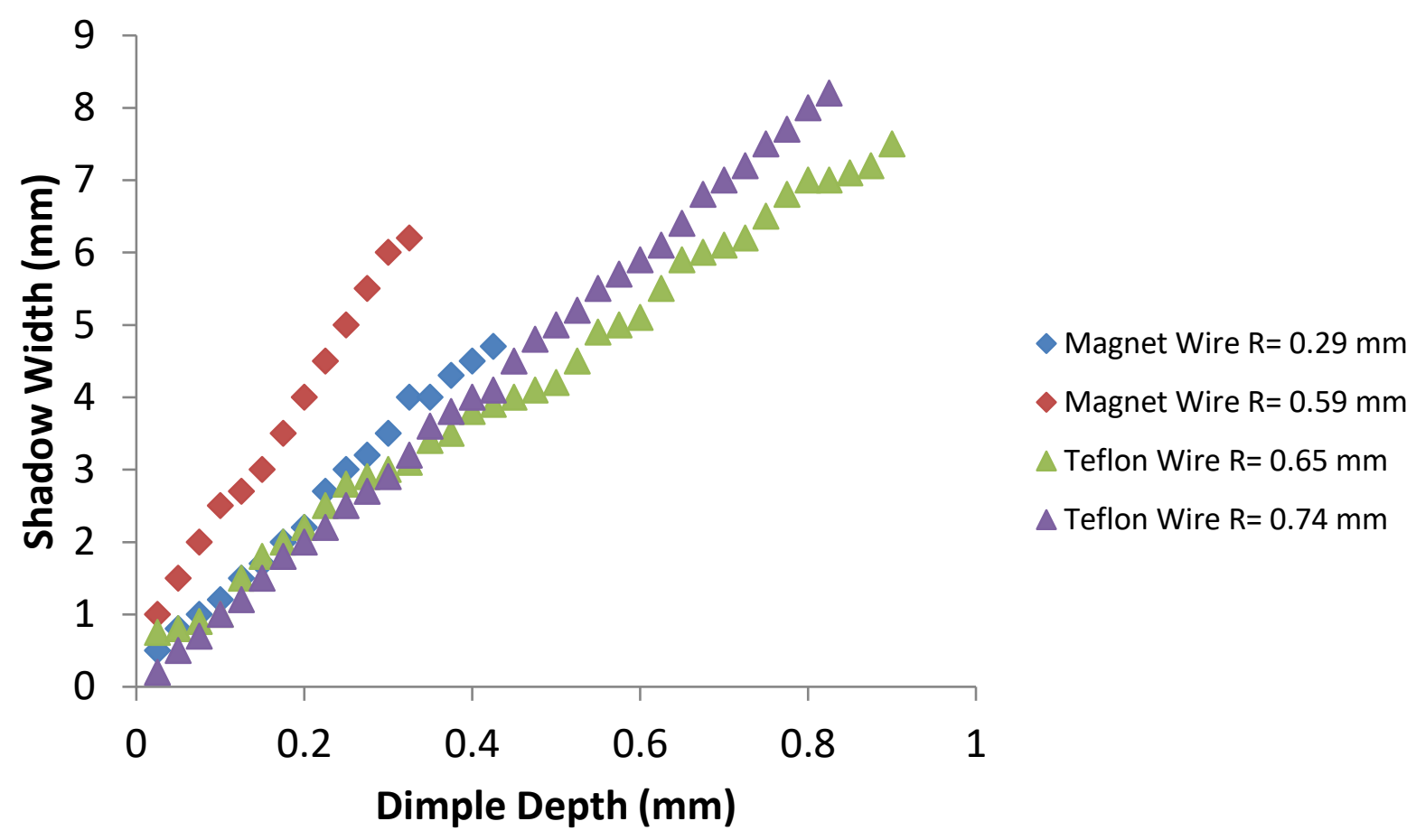

Fig. 9: Shadow width vs. dimple depth for magnet and Teflon wires with varying radii.
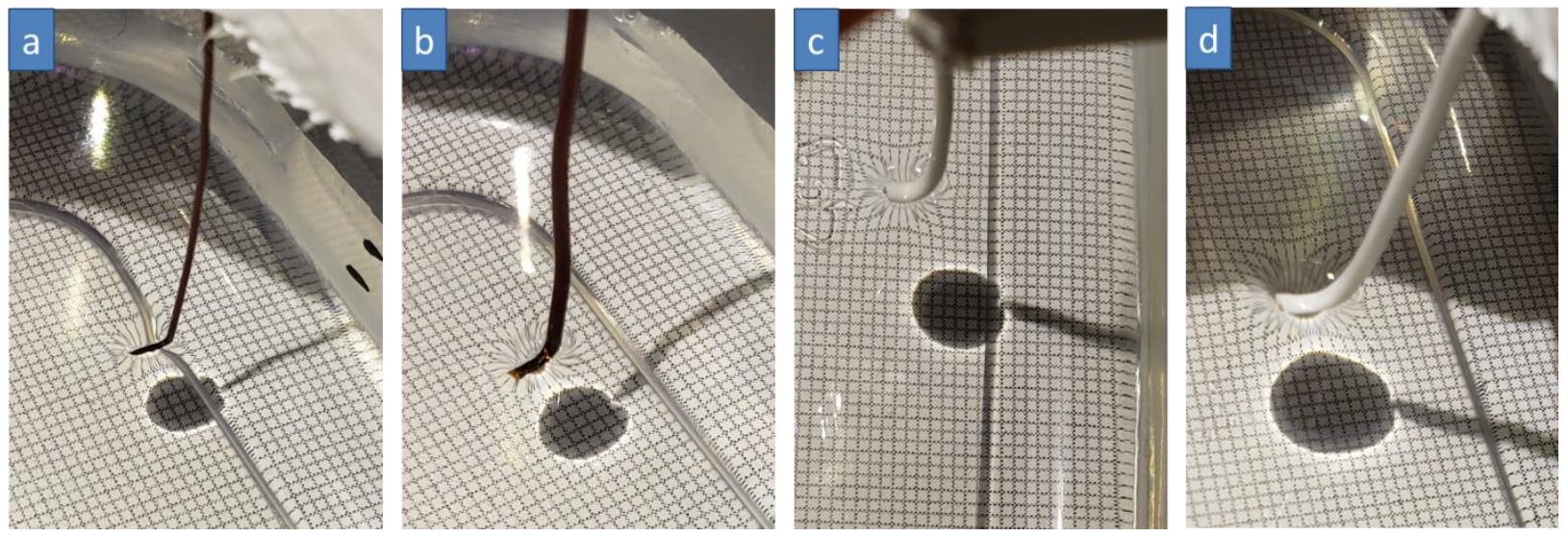

Fig. 10: Maximum shadow widths for the a) $0.29 \mathrm{~mm}$ radius magnet wire, b) 0.59 radius magnet wire, c) $0.65 \mathrm{~mm}$ radius Teflon wire, and d) $0.74 \mathrm{~mm}$ radius Teflon wire.

In order to examine the relationship between the wire radius, dimple depth, and shadow size, magnet wires of radius $0.29 \mathrm{~mm}$ and $0.59 \mathrm{~mm}$, and Teflon wires of radius $0.65 \mathrm{~mm}$ and $0.74 \mathrm{~mm}$ were used, with a water depth of $15 \mathrm{~mm}$ and a $15 \mathrm{~cm}$ distance from the light source to the water's surface. It was found that wires with a larger radius were able to penetrate the water surface more deeply, and as a result, create larger shadows (See Figs. 9 and 10). 
If this particular experiment were to be implemented in a physics lab, it is recommended that a wider range of wire gauges be used; it was the unfortunate case that at the time of this writing, only two different wire gauges were available for the two different materials. A wider variety of gauges will provide a greater abundance of data from which the experimenter can draw more concrete conclusions than what is provided in this work. Moreover, magnet wire is not generally recommended as a material in an experiment such as this, as obtaining the contact angle of the wire's coating is a point of difficulty. The composition of the coating of magnet wire generally varies from manufacturer to manufacturer, and the exact coating used is often difficult to ascertain due to the proprietary nature of the industry. However, in this work, it was useful to use the magnet wire in order to attain a qualitative understanding of the relationship between wire radius, dimple depth, and shadow size.

\section{Water Depth}

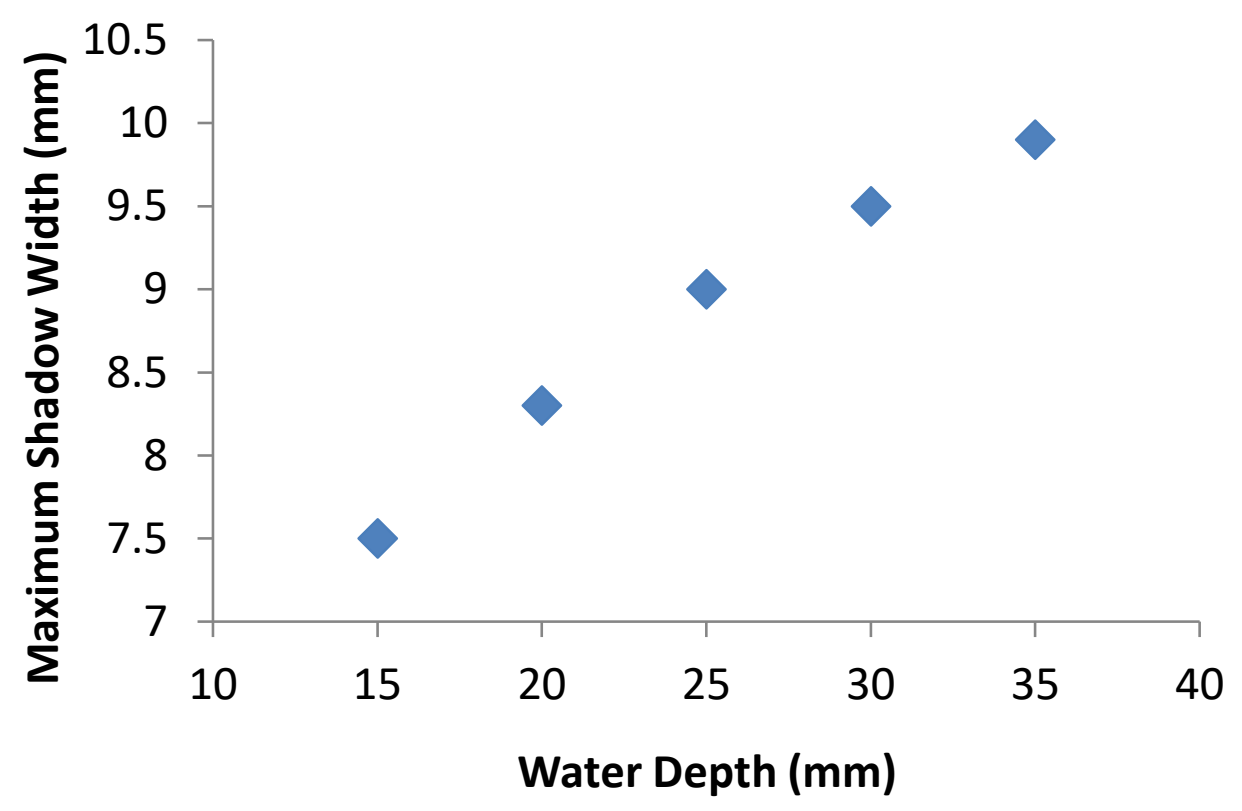

Fig. 11: Maximum shadow width vs. water depth.
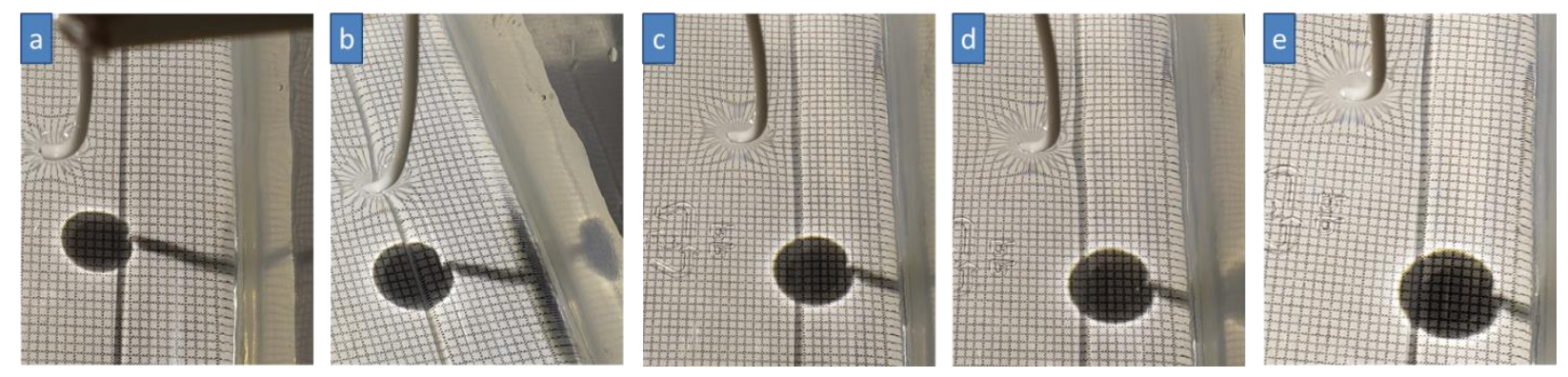

Fig. 12: Maximum shadow widths observed for water depths of a) $15 \mathrm{~mm}$, b) $20 \mathrm{~mm}$, c) $25 \mathrm{~mm}$, d) 30 $\mathrm{mm}$, and e) $35 \mathrm{~mm}$.

Equation 14 predicts that shadow widths should generally increase linearly with increasing water depth $\left(H_{w}\right)$, which was experimentally observed to be the case (see Figs. 11 and 12). Using a $0.65 \mathrm{~mm}$ 
radius Teflon wire, the smallest shadow width of $7.5 \mathrm{~mm}$ was observed at a water depth of $15 \mathrm{~mm}$, while the largest was observed to be $9.9 \mathrm{~mm}$ at a water depth of $35 \mathrm{~mm}$.

\section{Various Fluids}

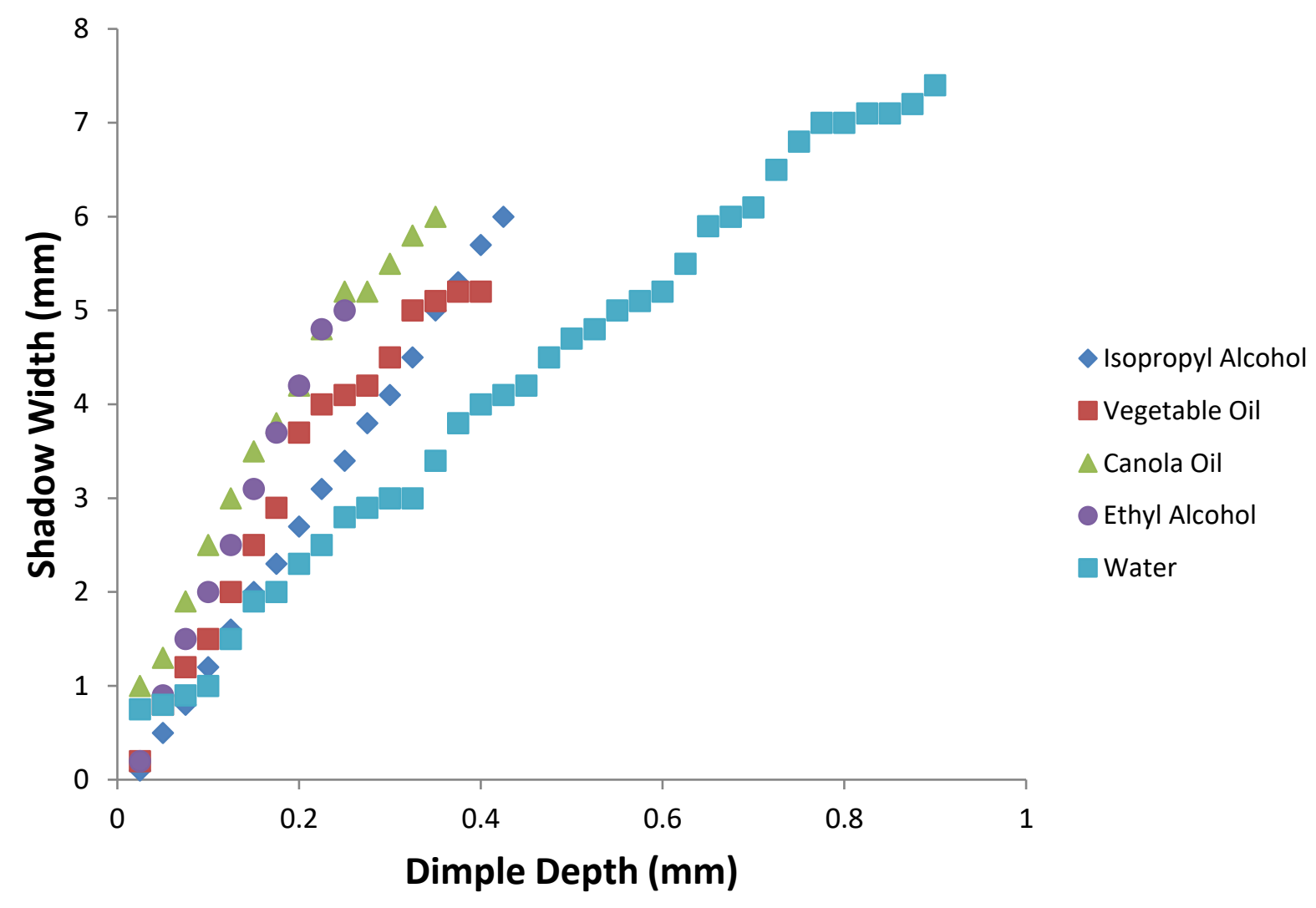

Fig. 13: Shadow width vs. dimple depth for fluids of varying refractive index and surface tension, using the $0.65 \mathrm{~mm}$ Teflon wire.
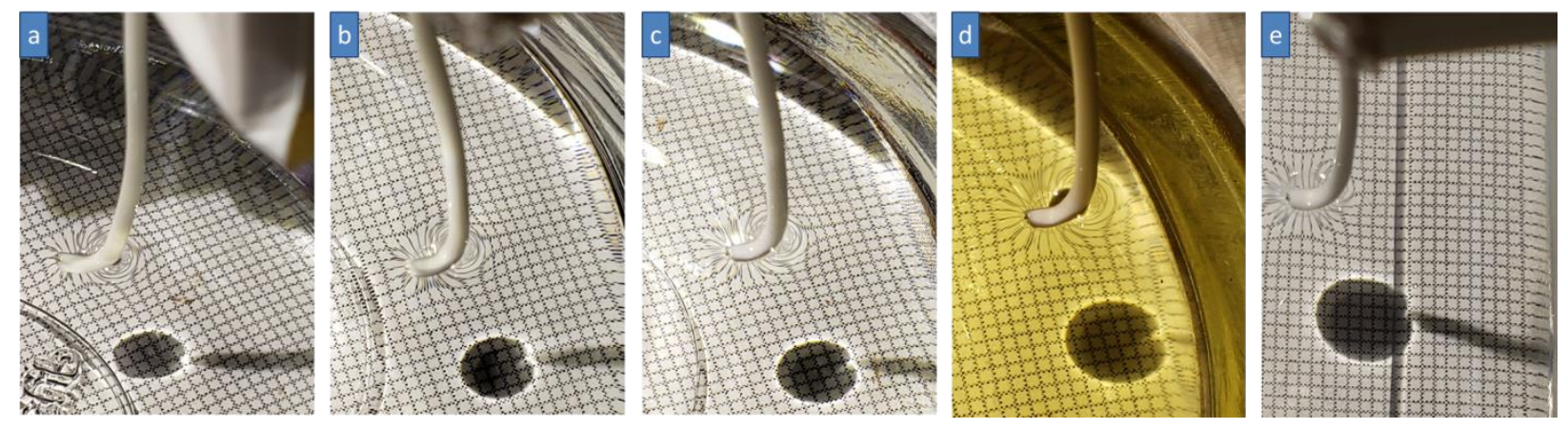

Fig. 14: Maximum shadow widths observed using the $0.65 \mathrm{~mm}$ Teflon wire and various $15 \mathrm{~mm}$-deep fluids: a) ethyl alcohol, b) isopropyl alcohol, c) vegetable oil, d) canola oil, and e) water.

The relationships between refractive index and shadow size, and surface tension and dimple depth were examined by using fluids of varying refractive index and surface tension. The fluids used in this 
work were: ethyl alcohol $(n=1.362, \sigma=22.3 \mathrm{mN} / \mathrm{m})$, isopropyl alcohol $(n=1.377, \sigma=23.0 \mathrm{mN} / \mathrm{m})$, vegetable oil $(n=1.470, \sigma=31.3 \mathrm{mN} / \mathrm{m})$, canola oil $(n=1.465, \sigma=31.3 \mathrm{mN} / \mathrm{m})$, and water $(n=1.334, \sigma$ $=72.8 \mathrm{mN} / \mathrm{m}$ ). ${ }^{8,9,10,11,12}$ It was found that water, having the highest surface tension by far, was able to be used to create much deeper dimples than the other fluids used, resulting in much larger shadows, despite having the smallest refractive index of all the materials (see Figs. 13 and 14). Ethyl alcohol, on the other hand, created the smallest shadows and shallowest dimples due to its low surface tension and only slightly larger refractive index in comparison to that of water. Perplexingly disparate results were obtained for vegetable oil and canola oil, despite their similar refractive indices and surface tensions, with vegetable oil creating the shallower dimples and smaller shadows of the two materials. These results suggest that it is possible that there is another variable that has thus far gone unaccounted for in this work that can affect the dimple depth (and thus, shadow size), and further studies may seek to identify such a variable. However, based on these overall results, it appears that the general trend is that the shadow size is largely governed by the surface tension of the fluid used, as the dimple depth seems to have a larger effect on shadow size than does the refractive index of the fluid.

\section{Applications}

The methods used in this paper can be used to create a lab assignment for physics students to explore geometrical optics using a commonly observed real world phenomenon. The setup pictured in Fig. 4 is inexpensive, costing under $\$ 50$ per setup if one uses preexisting ring stands frequently available in most physics classrooms. Some possible lab activities related to this phenomenon could be: experimental measurement of contact angles for different materials, a comparison of dimple depth and shadow width for materials with different contact angles, a comparison of shadow sizes at different water depths and/or distances between the LED lamp and the water surface, a comparison of dimple depth and shadow width for fluids with different indices of refraction and surface tension, a comparison of dimple depth and shadow width for various leg radii, drawing a ray diagram of the incident and refracted rays on the water surface by hand, as well as computational modeling of the water surface and resultant shadows as performed in this work. The goal for such a lab assignment would be to improve student understanding of geometrical optics, with respect to lenses and Snell's law, as well as the physics of interactions between a fluid and solid at the air-fluid-solid interface, with respect to surface tension, buoyancy force, and contact angles. Because of the complexity of the equations required to model the water's surface, this type of lab is well-suited for a computationally oriented introductory physics course. ${ }^{13}$ It is also wellsuited for an intermediate level experimental physics class.

\section{CONCLUSIONS}

In this work, the deformation of a fluid's surface due to a water-walking insect's leg, as well as the shadows resulting from the formation of a fluidic lens were discussed. An inexpensive experimental setup was created to emulate the conditions under which these shadows are formed in a controlled environment, using an LED lamp and a 3D printed apparatus that raised and lowered wires of varying radius and composition on to a water surface. The methods used in this work can be used in a physics laboratory environment to help students to better understand geometrical optics with respect to lenses and Snell's law, as well as the interactions between fluids, solids, and air with respect to buoyancy force, surface tension, and contact angles.

\section{ACKNOWLEDGMENTS}


The authors would like to thank Zachary Dale of the Portland State University Physics Department for his contribution in designing and printing the 3D printed height apparatus used in this work. This work was performed under the auspices of the Portland State University Physics Department. 


\section{REFERENCES}

1 Yun Seong Song and M. Sitti, IEEE Transactions on Robotics 23, (2007).

2 X. Zhang, J. Zhao, Q. Zhu, N. Chen, M. Zhang and Q. Pan, ACS Applied Materials \& Interfaces 3, (2011).

3 P. Wei, S. Chen and J. Lin, Langmuir 25, (2009).

4 W. Yin, Y. Zheng, H. Lu, X. Zhang and Y. Tian, Applied Physics Letters 109, (2016).

5 Y. Zheng, H. Lu, W. Yin, D. Tao, L. Shi and Y. Tian, Langmuir 32, (2016).

6 Y.Song, S. Suhr, M. Sitti, IEEE International Conference on Robotics and Automation, 2303, (2006).

7P. Moran, S. Dharmatilleke, A. Khaw, K. Tan, M. Chan and I. Rodriguez, Applied Physics Letters 88, (2006).

8 Surface Tension: Liquids Stick Together.

http://labsci.stanford.edu/images/Surface-Tension-TA.pdf

9 Przybylski, R.

https://fr.canolacouncil.org/media/515239/canola_oil_physical_chemical_properties_1.pdf

10 S. Sahasrabudhe, V. Rodriguez-Martinez, M. O’Meara and B. Farkas, International Journal of Food Properties (2017).

11 Surface Tension of Ethanol.

http://www.ddbst.com/en/EED/PCP/SFT_C11.php

12 Reference Table: Refractive Indices of Known Materials and Brix Scale.

http://www.raeco.com/training/refractive-index-values.htm

13 R. Chabay and B. Sherwood, American Journal of Physics 76, (2008). 